\title{
FRAGTIONAL ITERATION NEAR A FIXPOINT OF MULTIPLIER 1.
}

\author{
I. N. BAKER
}

(received 28 November 1963)

\section{Introduction}

An analytic function $f(z)$ is said to have a fixpoint $\xi \neq \infty$ of multiplier I if $f(\xi)=\xi, f^{\prime}(\xi)=1$. The function then has an expansion

$$
f(z)=\xi+(z-\xi)+\sum_{m+1}^{\infty} a_{k}(z-\xi)^{k}, a_{m+1} \neq 0, m \geqq 1 .
$$

It has been shown in [1] that there is for every complex $s$ a unique formal iterate

$$
f_{o}(z)=\xi+(z-\xi)+\sum_{m+1}^{\infty} a_{k}(s)(z-\xi)^{k}, a_{m+1}(s)=s a_{m+1},
$$

(where the $a_{k}(s)$ are well-defined polynomials in $s$ ) satisfying the formal identity

$$
f \circ f_{s}(z)=f_{8} \circ f(z)
$$

(where $f \circ g(z)$ denotes $f(g(z))$ ) and indeed

$$
f_{s} \circ f_{t}(z)=f_{s+t}(z) \text {. }
$$

The series $f_{1}(z)$ is identical with $f(z)$ and, more generally, for $s=n$, a positive integer, $f_{n}(z)$ is identical with the formal iterate $f \circ f \circ \cdots \circ f(z)$; by analogy the $f_{s}(z)$ are called fractional iterates.

It was shown in [1] that the set of $s$-values corresponding to $f_{s}$ with a non-zero radius of convergence has one of the forms: (i) the whole complex $s$-plane, (ii) a discrete one-dimensional lattice $\left\{n s_{1}\right\}, n=0, \pm 1, \cdots, s_{1} \neq 0$ or (iii) a discrete two-dimensional lattice $\left\{m s_{1}+n s_{2}\right\}, m, n=0, \pm 1, \cdots, s_{1} / s_{2}$ not real. Cases (i) and (ii) were shown to occur for $f=z /(1-z)$ and $t=e^{z}-1$ respectively. G. Szekeres [4] has shown

THEOREM 1. If $f(z)$ in (1) is an entire or rational function and $\mathbb{S}$ is the set of those s-values for which the series (2) has a positive radius of convergence, 
then $\subseteq$ is not the whole plane (and consequently $\subseteq$ is a discrete lattice) except in the case

$$
f(z)=\xi+(z-\xi) /\{1+a(z-\xi)\},
$$

when

$$
f_{s}(z)=\xi+(z-\xi) /\{1+a s(z-\xi)\} .
$$

In this connexion Szekeres asks whether the same result holds for the class of meromorphic or, more generally, single-valued analytic functions. We shall prove:

THEOREM 2. If (1) is the expansion about $\xi$ of a meromorphic function, then $\sqrt{5}$ is the whole plane only when the function has the form (5).

The proof of 2 will give a somewhat different approach to Theorem 1. It may easily be shown (c.f. [1, section 7$],[4$, introduction]) that there are series (1) other than (5) for which $\subseteq$ is the whole plane: these examples of functions of $f(z)$ and their iterates are many-valued under analytic continuation. It may be remarked in conclusion that no examples are known where $\subseteq$ is a two-dimensional discrete lattice.

\section{Preliminary results}

We shall prove Theorem 2 only for the case $m=1$ in (1), i.e. when

$$
f(z)=\xi+(z-\xi)+a_{2}(z-\xi)^{2}+\sum_{3}^{\infty} a_{k}(z-\xi)^{k}, \quad a_{2} \neq 0 .
$$

The ideas in the general case are the same, but the description of certain regions involved is more complicated.

It is convenient to transfer the fixpoint $\xi$ to $\infty$. If we change variables in the transformation $z_{1}=f(z)$ by putting $z-\xi=k / t, z_{1}-\xi=k / t_{1}$, the function $f(z)$ becomes for suitably chosen $k$ (c.f. [4])

$$
t_{1}=g(t)=t+1+\sum_{1}^{\infty} b_{k} t^{-k}
$$

and the same change of variables turns $f_{s}(z)$ into

$$
t_{s}=g_{s}(t)=t+s+\sum_{1}^{\infty} b_{k}(s) t^{-k}
$$

The $g_{s}$ are the unique series of form (9) such that

$$
g_{s} \circ g=g \circ g_{s}
$$

further 


$$
g_{8} \circ g_{t}=g_{s+t},
$$

and $g_{s}(t)$ is convergent for values other than $t=\infty$ if and only if $f_{\mathrm{s}}$ in (2) is convergent for values other than $z=\xi$.

From now on $g_{8}(t)$ will denote the series (9) and $g=g_{1}$ will be assumed convergent for $|t|>R$.

We quote the following results from $[1,2]$ :

LEMMA 1 [1, p. 272] If the region $\mathfrak{D}(K)=\bigcup_{-(\pi / 4) \leqq \alpha \leqq(\pi / 4)} \mathfrak{E}(\alpha, K)$, where $\mathcal{E}(\alpha, K)$ is the half-plane $\left\{z \mid \operatorname{Re}\left(z e^{-i \alpha}\right)>K\right\}$, then for all sufficiently large $K(>R), g_{n}(z)$ is regular,

$$
g_{n}(z) \in \mathfrak{D}(K), \quad n=1,2, \cdots
$$

and

$$
\operatorname{Re} g_{n}(z) \rightarrow \infty \text { as } n \rightarrow \infty
$$

for all $z$ in the closure $\overline{\mathfrak{D}}(K)$ of $\mathfrak{D}(K)$.

By $[1,273(21)](13)$ holds uniformly on any compact subset of $\mathfrak{D}(K)$.

LEMMA 2 [1, p. 273] For all sufficiently large $K$ the domain $\mathscr{D}(K)$ of lemma 1 has the properties:

$$
A(t)=\lim _{n \rightarrow \infty}\left\{g_{n}(t)-n-b_{1} \log n\right\},
$$

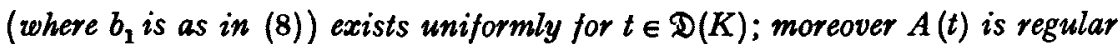
and schlicht in $\mathfrak{D}(K)$ and $A^{\prime}(t) \rightarrow 1$ uniformly as $t \rightarrow \infty$ in $\mathfrak{D}(K)$. One has

$$
A\left(g_{n}(t)\right)=A(t)+n \text { for } t \in \mathfrak{D}(K) .
$$

Lемма $3[4, \S 2]$ If the series (9) have a positive radius of convergence for every $s$, then $b(t)=A^{\prime}(t)$ is regular in a full neighbourhood of $t=\infty$ and has an expansion

$$
b(t)=1-b_{1} t^{-1}+\sum_{2}^{\infty} \beta_{k} t^{-k}
$$

which may be calculated from

$$
b \circ g(t)=b(t) / g^{\prime}(t) .
$$

We now prove

Lемма 4. If the series (9) have a positive radius of convergence for every $s$, and if $g_{n}(t)$ are single-valued in their whole domain of existence for $n=1,2, \cdots$, then there exists $R_{0}>0$, such that for $t$ in any annulus $R_{0}<R_{1} \leqq|t| \leqq R_{2}<\infty$ one has for all large enough $n$ 
(i) $g_{n}(t)$ regular, $g_{n}(t) \in \mathfrak{D}(K)$, and

(ii) $g_{n}(t) \rightarrow \infty$ uniformly as $n \rightarrow \infty$.

Proof. Choose $K$ so large that Lemma 2 holds and that [Lemma 3] $A^{\prime}(t)$ is regular in $|t|>K$. By enlarging $K$ we may suppose $A^{\prime}(t)$ as close to 1 (uniformly) as we please in $\mathfrak{D}(K)$. Then $t \rightarrow \omega=A(t)$ maps $\mathfrak{D}(K)$ univalently and conformally on to a region $\mathbb{E}$ of the $\omega$-plane lying to the right of a curve (of the same general appearance as the boundary of $\mathscr{D}(K)$ ) which approaches $\infty$ in the directions arg $\omega= \pm 3 \pi / 4$. $\odot$ contains a half plane $\operatorname{Re} \omega>B$. One may now take $R_{0}>K, R_{0}<R_{1}<R_{2}$. Let $R_{1}<r<R_{2}$ and $\gamma$ be the segment $t>r$, of the real $t$ axis, $\beta$ the semicircle $t=r e^{i \theta}, 0 \leqq \theta \leqq \pi$. By Lemma $3, b(t)=A^{\prime}(t)$ is regular on $\beta \cup \gamma$, and so $A(t)$ may be continued regularly along $\beta \cup \gamma$ to $t=-r$, the values $A(\beta)$ being bounded. For all large enough $n$ the values $A(\beta)+n$ lie in the half plane $\operatorname{Re} \omega>B$, while for all positive $n, t \in \gamma \subset D(K)$ implies by (15) $A(t)+n=A\left(g_{n}(t)\right) \in \mathbb{E}$. Thus $A(\beta \cup \gamma)+n$ is a curve in $\mathbb{E}$. Consider $A_{-1}\{A(t)+n\}=h(t)$ on $\beta \cup \gamma$. On $\gamma, h(t)=g_{n}(t)$, while as $t$ describes $\beta \cup \gamma, A(t)+n$ describes $A(\beta \cup \gamma)+n$ in $\mathbb{E}$ and the inverse of the schlicht map $A: \mathfrak{D}(K) \rightarrow \mathbb{C}$ gives a regular continuation $h(t)$ of $g_{n}(t)$ along $\beta$ to $-r$. Moreover for $t=r e^{i \theta}, 0 \leqq \theta \leqq \pi, R_{1} \leqq r \leqq R_{2}$ we have $g_{n}(t)$ lying in a compact subset of $\mathscr{D}(K)$.

A similar argument may be applied to the path $\beta^{\prime}+\gamma$ where $\beta^{\prime}: r e^{2 \theta}$, $0 \geqq \theta \geqq-\pi$. The assumption that $g_{n}(t)$ is single-valued assures that both the upper and lower continuation yield the same result for $g_{n}(t)$. Thus $g_{n}(t)$ is regular in the annulus $R_{1} \leqq|t| \leqq R_{2}, g_{n}(t)$ maps the annulus into a compact subset of $\mathfrak{D}(K)$, and by lemma 1 (ii) follows.

We may restate lemma 4 in terms of the function $f(z)$ with a finite fixpoint $\xi$ :

\section{Lemma 5. If the series (7)}

$$
f(z)=\xi+(z-\xi)+a_{2}(z-\xi)^{2}+\sum_{3}^{\infty} a_{k}(z-\xi)^{k}, \quad a_{2} \neq 0,
$$

and the fractional iterates (2)

$$
f_{s}(z)=\xi+(z-\xi)+s a_{2}(z-\xi)^{2}+\sum_{3}^{\infty} a_{k}(s)(z-\xi)^{k}
$$

all have a positive radius of convergence, and if $f_{n}(t)$ are single valued in their whole domain of existence for $n=1,2, \cdots$, then there exists $\rho_{0}>0$, such that for $z$ in any annulus $0<\rho_{2} \leqq|z-\xi| \leqq \rho_{1}<\rho_{0}$, one has for all large enough $n$

(i) $f_{n}(z)$ regular, and

(ii) $f_{n}(z) \rightarrow \xi$ uniformly as $n \rightarrow \infty$. 


\section{Proof of theorem 2}

Without loss of generality we assume $\xi=0$. Suppose that $f(z)$ is entire or meromorphic and that the set $\subseteq$ of theorem 2 is the whole plane.

(i) In the case where $f(z)$ is rational or entire the theorem follows at once from results of Fatou [2,3] who proved that (i) a fixpoint of multiplier 1 belongs to the set $\mathfrak{F}$ of nonnormality of $\left\{f_{n}(z)\right\}$, (ii) $\mathfrak{F}$ is perfect (except when $f(z)$ is of the form (5)). Thus, however small we take $\rho_{1}$, there are points of $\mathfrak{F}$ in $0<|z| \leqq \rho_{1}$, and hence there is an annulus $0<p_{2} \leqq|z| \leqq p_{1}$ in which $\left\{f_{n}(z)\right\}$ is not a normal family, in contradiction to Lemma 5 .

(ii) If $f(z)$ is a transcendental meromorphic function we take $\rho_{0}$ as in Lemma 5 and show that there are in the disc $\Re:|z|<\rho_{0}$ no antecedents of poles of $f(z)$, i.e. no $\beta$ for which $\alpha=f_{n}(\beta)$ is a pole of $f(z)$. If $\beta$ were such a point, then for any neighbourhood $\mathfrak{N}$ of $\beta, f_{n}(\mathfrak{R})$ is a neighbourhood of $\alpha, f_{n+1}(\mathfrak{R})$ a neighbourhood of $\infty$, and $f_{n+k}(\mathfrak{R}), k \geqq 2$ includes all points of the plane with at most two exceptions. Thus $\beta$ is an essential singularity of all $f_{n+k}(z)$ in contradiction to lemma 5 . Thus our assertion is established and it follows that all $f_{n}(z)$ are regular in $\Re$.

We now note that $\left\{f_{n}(z)\right\}$ does not form a normal family in $\Re$. If $\left\{f_{n}(z)\right\}$ is normal, then we can extract a subsequence $\left\{f_{n^{\prime}}(z)\right\}$ uniformly convergent in $|z| \leqq \rho_{1}<\rho_{0}$ to a regular function, which by Lemma 5 is identically zero. But for this it is necessary that $f_{n^{\prime}}^{\prime}(0) \rightarrow 0$, while, in fact $f_{n^{\prime}}^{\prime}(0)=1$.

Since $\left\{f_{n}\right\}$ is not normal in $\Re$ the functions $f_{n}(z)$ take in $\Re$ all values with at most two exceptions. If there are at least two (finite) poles $a, b$ then $t_{n}=a, b$ or $\infty$ in $\AA$, which contradicts the first paragraph of (ii). We are left only with the case when $f(z)$ has a single pole $a$. If $f(z)=a$ has a solution $c$, then $c \neq a$ and we can find solutions in $\Re$ of $f_{n}=c, a$ or $\infty$, which again gives a contradiction.

If $f(z)$ has a single pole $a$ and $f(z)=a$ has no solution \{e.g. $f(z)=$ $\left.a+e^{z} /(z-a)\right\}$, consider the meromorphic function

$$
\begin{gathered}
h(z)=(f(z)-z) /(f(z)-a), \\
f(z)=(a h(z)-z) /(h(z)-1) .
\end{gathered}
$$

The function $h(z)$ has no finite poles (since $f(z) \neq a$ ) and is not a polynomial since otherwise $f(z)$ would be rational by $\left(17^{\prime}\right)$. Hence $h(z)$ is entire transcendental and $h(z) \neq 1$ for $z \neq a$. Therefore by Picard's theorem it has infinitely many zeros and $f(z)=z$ has infinitely many solutions. Returning to the non-normal family $f_{n}(z)$ we see that there is a $z_{0} \in \mathfrak{I}$ such that $f_{n_{0}}\left(z_{0}\right)=z_{1}, z_{1} \neq 0, f\left(z_{1}\right)=z_{1}$. Then $f_{n}\left(z_{0}\right)=z_{1}$ for all $n>n_{0}$, and in any annulus $0<\rho_{2} \leqq|z| \leqq \rho_{1}<\rho_{0}$ containing $z_{0}$, the sequence $f_{n}(z)$ cannot tend uniformly to 0 .

We have now established theorem 2 in all cases. 


\section{Extensions}

The differences caused by assuming $m>1$ in (1) are that the region $\mathfrak{D}(K)$ of Lemmas 1,2 must be replaced by a set of $m$ smaller ones (essentially sectors of opening $3 \pi / 2 m$; c.f. [1]), and that the expansion (16) of $A^{\prime}(t)$ has a different form. We shall not state the necessary modifications in the proof of theorem 2.

It is interesting to note that our method extends to further classes of functions. If, for example, $f(z)$ in (1) is a single-valued function defined in the whole plane except for a number $(>2)$ of isolated essential singularities, then the argument of $\S 3$ (ii) shows that there are no antecedents of these singularities in a disc $\Re$ surrounding $z=\xi$ and the family $\left\{f_{n}\right\}$ is consequently regular and normal in $\Re$, which by the second paragraph of $\S 3$ (ii) cannot be the case.

Although I have been able to prove various results of the above type, I have not been able to extend theorem 2 to cover all single-valued $f(z)$.

\section{References}

[1] Baker, I. N., Permutable power series and regular iteration, J. Australian Math. Soc. 2 (1962), $265-294$.

[2] Fatou, P., Sur les équations fonctionnelles, Bull. Soc. Math. France 47, 161-271 (1919); $48,33-94,208-314(1920)$.

[3] Fatou, P., Sur l'itération des fonctions transcendantes entières, Acta Math. 47, 337-370 (1926).

[4] Szekeres, G., Fractional iteration of entire and rational functions, this Journal 4 (1964). 129-142.

Imperial College of Science and Technology, London. 